hilarity and movement (presumably including eye movement) during the compression phase due to the combined effects of warmth and nitrogen narcosis, thus assisting the flow of nitrogen-laden tears between the lens and cornea, while during decompression the chamber was, as is usual, rather cold and foggy, with the subjects relatively immobile, thus minimizing any flow of tears which might have helped to wash out the nitrogen. As the other eye was only slightly affected, it must be presumed that there was a difference in the fit, and hence in the flow of tears under the two lenses, causing either less absorption or better elimination of gas from the other eye.-I am, etc.

\section{JoHN BeTts.}

London N.10.

Chairman, National Medical Committee.

\section{Bowel Motility and Colonic Cancer}

SIR,-Recent studies ${ }^{1}$ and comments ${ }^{2}$ on cancer of the colon indicate that valuable information may be forthcoming from intensive studies of bowel motility and faeces composition in populations with contrasting prevalences of this type of cancer.

In South African Bantu age specific mortality is only a tenth of that in whites." In preliminary studies on this subject we have noted two points of interest. In the Bantu (childron and students studied thus far) a third have three motions daily compared with only $1-2 \%$ in whites. Moreover, in terms of exposure of bowel mucosa to digesta, carmine studies have revealed that in the Bantu the mean transit time of the longest third is less than the mean time of the quickest third among whites. The finding to be stressed is that even in Bantu and whites with the same frequency of defaecation (say, one to two motions per diem) traces of carmine continue to be seen far longer in stools of whites than in Bantu; in whites the period is sometimes five to seven days. In addition, therefore, to the presumably vulnerable constipated moiety of population, ${ }^{2}$ there is this further moiety with regular motions, not constipated, who, unknown to themselves and undetectable clinically, have unduly prolonged times of traversal of digesta. This is the condition which conceivably provides the favourable milieu intérieur required for carcinogenesis, whether of exogenous or endogenous origin.

The second finding to be emphasized concerns the differing ability to produce a stool quickly on request. Eighty to ninety per cent. of rural Bantu children have this capacity, 70-80\% of partially sophisticated urban Bantu, but fewer than $10 \%$ of white children. Previouslys ${ }^{5}$ we noted that when young white adults consumed a very high-residue Bantu-type of diet for several days they still could not produce a stool on request in the same facile manner as Bantu. Evidently, with "civilization," voluntary control of the defaecation reflex is largely lost. May not differences in physiological tone of the colon be associated with different susceptibilities to carcinogenic agents ?

The critical question, of course, is whether the moiety with protracted exposiure to possible carcinogens is in fact that moiety in which sufferers from bowel cancer are to be found. Elucidation of this problem will be difficult. For example, verbal questioning regarding bowel habits, etc., is often worthless; we find a poor correlation between frequency from recall and frequency by keeping a weekly chart. Further, not recollectable to patients, their bowel habits, etc., at 50-60 years may differ markedly from those at $30-50$ years.

Obviously, far more explicit data are needed on populations with contrasting prevalences of colonic cancer. In the present circumstances it would seem that only by impeding carcinogenic action may prophylaxis be envisaged. Screening centres have been advocated to detect the segment of population most prone to coronary heart disease, to detect the aglycosuric population who have abnormal glucose tolerance, etc. Would it be too far fetched to suggest that the younger adult population be screened, initially with carmine, to detect the moiety with very prolonged transit times, and that such be given dietary or therapeutic advice to accelerate excretion ?-We are, etc.,

Alexander R. P. Walker. B. FAITH WALKER.

\section{South African Institute for
Medical Research,} hannesburg,

Johannesburg,

\section{REFERENCES}

Wynder, E. L., and Shigemetsu, Cancer (Phila delphia), 1967,20,

3 Oettle. A. G., fournal of the National Cancer Institute, 1964, 33, 383.
Higginson, Journal of the National Cancer Walker, A. R. P., Nature, 1949, 164, 825

Walker, A. R. P., unpublished work.

\section{Pigmentation in Megaloblastic Anaemia}

SIR,-We have read with interest the description by Dr. N. Baumslag and Dr. J. Metz of spotty pigmentation of palms, soles, and tongue in lactating Africans with folate deficiency (21 June, p. 737). Clinicians in Ibadan are aware of the possibility of hyperpigmentation in patients with megaloblastic anaemia, as it has been seen in one Nigerian with vitamin $\mathbf{B}_{12}$ deficiency, but it has no been noticed in folate deficiency, thoug about 200 women with severe megaloblastic anaemia associated with pregnancy are seen each year. No areas of hyperpigmentation were revealed by a detailed dermatologica examination of six women with profound anaemia in pregnancy.

Failure to observe pigmentation in Ibadan may be because it has not been looked fo closely enough, or it may be related to the different presentation of folate deficiencyanaemia is seen most commonly near the end of the second trimester, while the South African patients were seen during lactation The South Africans may have been deficien for longer than the Nigerians, and shown an early stage of a skin lesion which is much more remarkable in vitamin $\mathrm{B}_{12}$ deficiency, ${ }^{1}$ a condition which develops insidiously and is almost always long-standing at the time of presentation.

Biopterin is the coenzyme required for hydroxylation of phenylalanine to tyrosine, and it has been found in abnormally high concentration in the serum of folate-deficien pregnant Nigerians. ${ }^{3}$ Widespread disturbance of amino-acid metabolism has been found in pregnant Nigerians with megaloblastic anaemia, but no evidence of a failure to convert phenylalanine to tyrosine (Jacobs, $\mathrm{S}$. and Fleming, A. F., unpublished observations). There is a lower than normal histidine $\alpha$-deaminase activity in the skin from vitamin
$\mathrm{B}_{12}$ or folate-deficient Indians with hyperpigmentation. ${ }^{4}$ None of these abnormalities of amino-acid metabolism help us to understand the accumulation of melanin in megaloblastic anaemia. A possible explanation is that it is the result of abnormal cell division. -We are, etc.

A. F. Fleming.

Department of Obstetrics and Gynaecology, University of Western Australia,

Skin Department,

Dryburn Hospital, REPERENCES

Watson-Williams, E. J., and Fleming, A. F.,
Blood, 1966, 28, 770.,

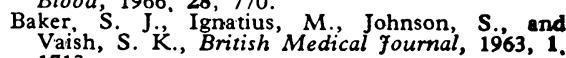
1713.

Fleming, A. F. and Broquist, H. P., American
fournal of Clinical Nurtition, $1967,20,613$ S. J., Clinica Chimica Acta, 1968, 20, 53.

\section{Hypokalaemia after Treatment with Duogastrone (Carbenoxolone)}

SIR,-Dr. J. Forshaw's Memorandum on hypokalaemic muscle weakness complicating the use of Duogastrone (position-release carbenoxolone sodium) (14 June, p. 674) prompts me to record a similar case.

A woman of 55 developed pins and needles in the fingers of both hands while cooking one evening. This lasted only 30 minutes, but an hour later she noticed weakness of both hands and the next day her right arm was weak. Over the next 24 hours the weakness spread to both arms, the shoulders, and then to the thighs and back muscles and she had difficulty in holdin up her head. She had no muscle pain, no diffculty in swallowing, and no visual symptoms. Her general health was good and there had been no diarrhoea or vomiting. She had never suffered from muscle weakness before, nor was there any family history of a similar condition. A duodenal ulcer had been demonstrated 23 years previously, but had always responded to conservative treatment. She had been taking Duogastrone, $200 \mathrm{mg}$. four times a day, for six weeks; the course of tablets had finished one day before the onset of her present symptoms.

On examination she was a fit-looking woman General examination was negative except for blood pressure which varied between $200 / 120$ and $140 / 80$, and a small nodule in the thyroid gland. There was slight weakness of jaw opening and weakness of the sternomastoids so that she had difficulty in lifting the head from the pillow. She had severe flaccid weakness in the limbs affecting proximal muscles more than distal and the arms more than the legs. There was no fasciculation or muscle tenderness, and the tendon reflexes were absent in the arm and at the ankles. The plantar responses were flexor. There was no impairment of sensation and the peripheral nerves were not thickened.

The relevant investigations were as follows : Serum electrolytes (mEq/1.): $\mathrm{Na} 149, \mathrm{~K} \mathrm{1.7,} \mathrm{Cl}$ $101, \mathrm{CO}_{2}$-combining power 32 . Serum enzymes (units): Aldolase 160, S.G.O.T. 244, S.G.P.T. 200. Glucose tolerance test $(30$ minute intervals following the ingestion of $50 \mathrm{~g}$. glucose): 117 $162,175,206,209 \mathrm{mg} . / 100 \mathrm{ml}$.

Electromyogram (right deltoid): mean muscle action potential duration $10 \mathrm{msec}$. (normal $14 \cdot 3$ $\pm 20 \%$ ) and incidence of polyphasic potentials $16 \%$ (normal 3-12\%). Maximum effort showed a normal interference pattern of reduced voltage. Conduction velocities in the ulnar and lateral popliteal nerves were within normal limits. These results strongly suggested a myopathic lesion (Dr. J. S. Chopra). Electrocardiograth : depression of ST segment in leads 1, 2, AVL V5, and V6: QT interval $0.4 \mathrm{sec}$ : prominent $\mathrm{U}$ waves in chest leads. 
Other investigations were normal, including chest $x$-ray, cerebrospinal fluid, tests of thyroid function, intravenous pyelogram, urinary ketoand hydroxysteroids, urinary aldosterone and plasma cortisol levels. Urine electrolyte excretion was at all times within normal limits. There was no myoglobinuria, but this test was not done until two weeks after admission to hospital. Muscle biopsy (left deltoid) showed no abnormality apart from one small collection of lymphocytes (Dr. Ingrid Allen). Efforts to provoke muscle weakness by administering glucose and insulin were unsuccessful.

For the first few days after admission to hospital there was some spontaneous improvement in muscle power; treatment was then started with potassium chloride by mouth, 2 g. three times a day, and she was discharged after six weeks with only minimal residual weakness. The serum enzymes returned to normal within four weeks, the hypertension disappeared, and a repeat glucose tolerance test after three weeks was normal. Electromyography four months later still showed slight myopathic changes, but by this time clinical recovery was complete and the tendon reflexes had returned.

In this case special efforts were made to eliminate other causes of hypokalaemia (for example, familial periodic paralysis, hyperthyroidism, potassium-losing nephropathy, and hyperaldosteronism) and carbenoxolone seems to be the most likely cause for the syndrome. The occurrence of reversible hypertension and diminished glucose tolerance in the acute phase is compatible with the "steroid-like" action of this drug. Like Dr. Forshaw's case, the implication of the drug therapy which the patient had been receiving was not realized until some days after admission to hospital. It is also of interest that significant spontaneous improvement in muscle power had already commenced before treatment with oral potassium was started.-I am, etc.,

\section{Department of Neurology, Royal Victoria Hospital and Belfast.}

\section{Scanning Electronmicroscopy in Dermatology}

SIR,-The scarning electron microscope is can be of value in comparative studies of the a promising tool for the study of the surface skin and of its appendages. In this study morphology of biological specimens, ${ }^{1-4}$ and a number of observations are reported on the following specimens: normal and diseased human hairs, psoriatic nail, and molluscum contagiosum lesions of the skin. These specimens were chosen because their low water content minimizes shrinkage artifacts, which could occur during processing, since it is necessary to examine the tissues under vacuum without prior embedding.

The specimens were air-dried, mounted on a Dural specimen holder, coated with approximately $500 \mathrm{~A}$ of evaporated gold/palladium alloy, and examined in the scanning electron microscope (Stereoscan Mk II, Cambridge Instrument Co. Ltd.).

The normal human hair showed a characteristic surface topography with well-defined individual scales (Fig, 1a). The surface aspects of the scales were more easily seen with the scanning electron microscope than with the usual replica techniques, and was of particular advantage in the study of diseased hairs (Fig. 1b). Similarly, modifications in the surface appearance of diseased nails were easily recognized, such as the characteristic surface pitting of the psoriatic nail (Fig. 2).

The great depth of focus of the scanning electron microscope allowed the whole specimen from the central cornified region of molluscum contagiosum lesions to be examined, providing a three-dimensional view of the inclusion bodies. These inclusion bodies consisted of hundreds of viral elements clumped together in an almost continuous array (Fig. 3). The viral elements

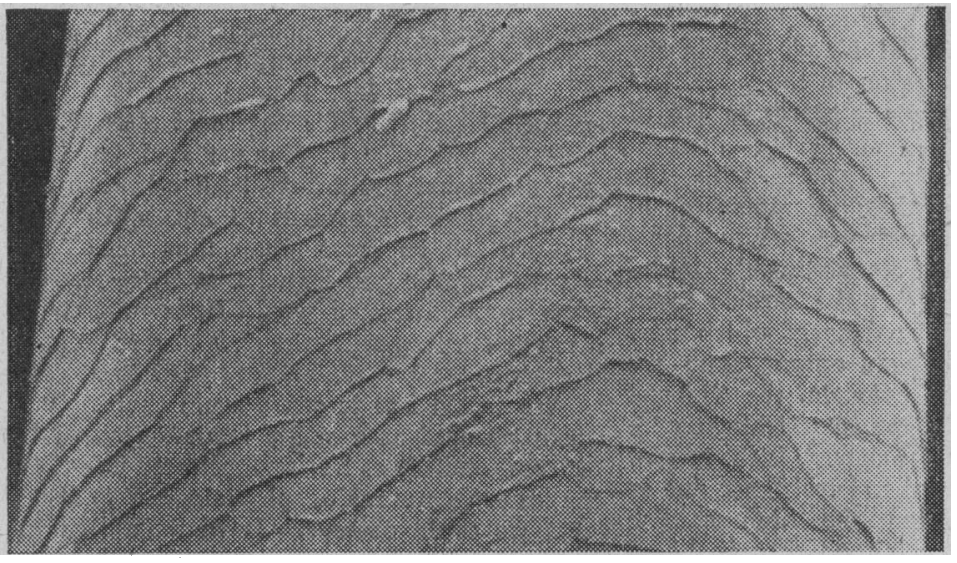

FIG. 1a.-Normal human hair showing clearly defined individual scales. $(\times 1,150$.

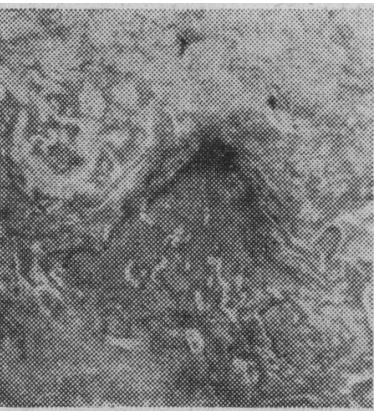

FIG. 2

FIG. 2.- Surface pitting of a psoriatic nail. $(\times 120$. Fig. 3. - Inclusion body
of molluscum contagiosum. $(\times 3,340$.

FIG. 4.-Viral elements in inclusion body of molluscum contagiosum showing surface details of particles. $(\times 10,920$.

FIG. 3

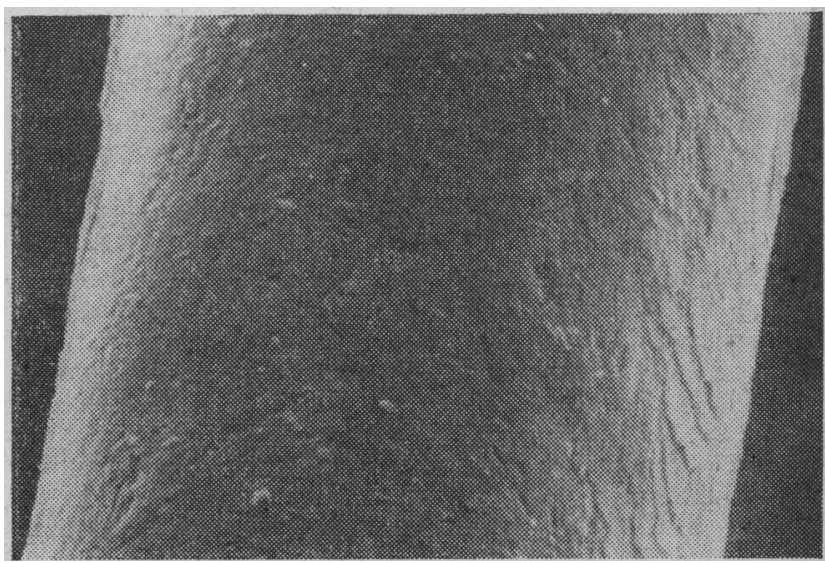

FIG. 1b.-Abnormal human hair showing loss of details of scales. $(\times 1,200$.)

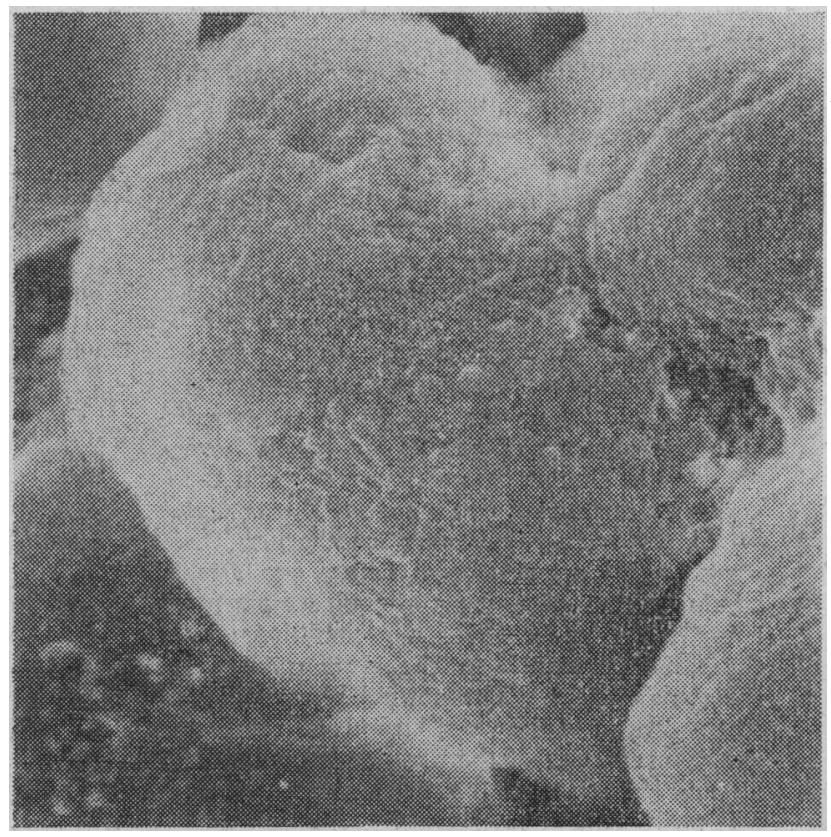

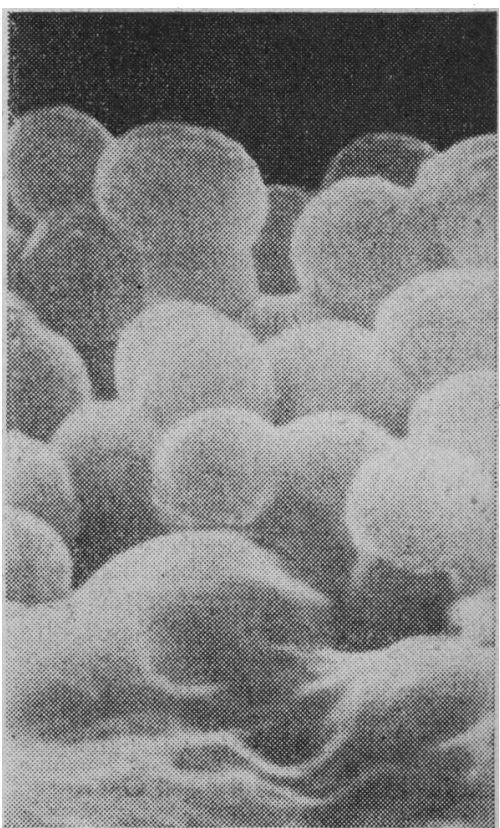

FIG. 4 